\title{
Quantum phase transitions and the extended coupled cluster method
}

\author{
N. E. Ligterink, ${ }^{*}$, N. R. Walet ${ }^{\dagger}$ and R. F. Bishop ${ }^{\ddagger}$ \\ Department of Physics, University of Manchester Institute of Science, P.O. Box 88, Manchester, M60 1QD, United Kingdom
}

(Received 4 August 2000; published 27 February 2001)

\begin{abstract}
We discuss the application of an extended version of the coupled cluster method to systems exhibiting a quantum phase transition. We use the lattice $O(4)$ nonlinear sigma model in $(1+1)$ and $(3+1)$ dimensions as an example. We show how simple predictions get modified, leading to the absence of a phase transition in $(1+1)$ dimensions, and strong indications for a phase transition in $(3+1)$ dimensions.
\end{abstract}

DOI: 10.1103/PhysRevE.63.037103

PACS number(s): 05.70.Fh, 11.30.Qc, 11.30.Rd, 64.60.-i

The nature of the ground-state wave function for (infinite) quantum systems is one of the underlying problems in many areas of physics, from atomic and condensed matter to highenergy physics. Special problems are encountered when one studies the transition between different ground states in cases where the balance of forces in a system with competing interactions is shifted. Since this is such a basic problem, a large number of calculational schemes exist to study the properties of the ground state. These schemes are either imaginary-time approaches, based on the fact that in imaginary time the state at lowest energy has the slowest rate of decay and can thus be filtered out, or are real-time schemes, usually based on the Rayleigh-Ritz variational principle. All of these methods have some weaknesses. In high-energy physics, for example, imaginary-time Monte Carlo calculations can only be performed on finite lattices. As a real phase transition can only occur in an infinite system, one has to find ways to extrapolate to infinite lattices, and hope no finite-lattice artifacts arise. Even if this can be done, an even more fundamental problem in the Monte Carlo integrations arises, due to the uncontrollable fluctuations introduced by fermionic degrees of freedom. This problem limits the validity of Monte Carlo approaches in condensed matter as well as those in lattice-(gauge)-field theory. Real-time methods, on the other hand, are usually based on rather complex many-body techniques, and lack the inherent simplicity of the implementations of Monte Carlo approaches. Also, even though most real-time methods start from the variational principle, this principle is often violated in an attempt to obtain a viable calculational scheme.

An important difference between real- and imaginarytime approaches is the way they deal with excitationswhereas in imaginary-time methods excited states need to be found by looking at exponentials with faster decay than the ground state, or via complicated Gramm-Schmidt orthogonalization procedures, the calculation of excitations is often much more straightforward for the real-time methods. Once we have constructed the ground state we can build excited states on top of this ground state, using sets of excitation

\footnotetext{
*Present address: ECT, Strada delle Tabarelle 286, I-38050 Villazzano (Tento) Italy. Electronic address: ligterin@ect.it

†Electronic address: n.r.walet@umist.ac.uk

¥Electronic address: r.f.bishop@umist.ac.uk
}

operators. The calculational scheme used is then very similar for both the ground state and the excited states.

In this Brief Report we shall concentrate on zero temperature, also called quantum, phase transitions. The most important exact statement for such situations is the Coleman no-go theorem [1] which states that, in one space dimension and under rather generous conditions, there is no phase transition to a state with long-range order. As is well known from the exact solution of the $X X Z$ model in $(1+1)$ dimensions [2], this does not preclude a phase transition with an apparent gapless mode, but without real long-range order. Notwithstanding such special cases, the theorem is a strong indictment against mean-field calculations, usually the lowest order in the real-time approaches, since these often predict phase transitions with long-range order even in one dimension. The reason is that mean-field theory ignores the strong interactions of the massless modes that accompany such a phase transition, which pushes up the mass of the would-be zero-mass modes.

One way to avoid this phenomenon is to concentrate on the phase with unbroken symmetry, and to construct a calculational scheme for that phase. Such approaches have been used in spin-lattice systems and in lattice field theories [3-6], but it has been found that they sometimes lead to termination points, probably indicating a phase transition, for the $(1+1)$-dimensional system as well as in higher dimensions. The excitation spectrum supports this behavior, and suggests a phase transition to a state with long-range order.

The most complicated part of the real-time approaches is the choice of a truncation scheme. One must make a selection which has enough degrees of freedom not to restrict the physical behavior of the system, nor to impose any assumptions of the nature of the phase transition on the system, and not too many to make the calculation infeasible. A good choice of wave function is key to any quantum many-body calculation, and more importantly, there must be a way to extend the calculations so as to test any assumptions being made.

Our approach is an example of such a formalism. The approach we use is, however, systematic and will not give rise to spurious phase transitions. The method is an offshoot of the coupled cluster method, called the extended coupled cluster method (ECCM) [7]. Theoretical aspects of the method have been analyzed in great detail in Ref. [7], but the number of applications to nontrivial models is extremely limited. It has several advantages over earlier versions. First 
of all, the mean-field approximation is a natural part of the hierarchy of approximations inherent to the method. It is thus suitable to deal with states at both sides of a phase transition, where one exists. We shall show in this letter that an (in principle infinite) set of improvements removes the spurious transition in one-dimensional systems. For the moment we shall not include the mean-field term in our calculations, but concentrate on two-site correlations instead. While this restricts us to the symmetric phase, it makes it easier to perform numerical calculations, which can be quite substantial.

We shall illustrate our method with a simple field theory, the $O(4)$ nonlinear sigma (NLS) model. We have chosen to discretize the Lagrangian for this model on a lattice in space, keeping time continuous. We then construct a Hamiltonian, which is the quantity being studied. The basic degrees of freedom are unitary two-by-two matrices, but these can also be represented by a four-dimensional unit vector $\left(\mathbf{n}, n_{4}\right)$, using a basis of Pauli matrices $\tau$,

$$
U=n_{4} I+i \tau \cdot \mathbf{n} .
$$

The Hamiltonian is most succinctly expressed in the angle between nearest-neighbor vectors, and the on-site generalized angular momenta,

$$
H=\frac{1}{2} \sum_{\mathbf{i}} \mathbf{I}_{\mathbf{i}}^{2}+\lambda \sum_{\langle\mathbf{i j}\rangle}\left(1-\cos \theta_{\mathbf{i j}}\right),
$$

where the sum over $\langle\mathbf{i j}\rangle$ runs over all nearest-neighbor pairs, and counts each pair (or lattice link) only once. The kinetic energy is proportional to the square of the (four-dimensional) angular momentum, $\mathbf{I}_{\mathbf{i}}^{2}$. This model shows great similarity with some Heisenberg-type models used in condensedmatter physics.

We expect a phase transition due to the competition between the kinetic and potential terms. For small $\lambda$, the weakcoupling limit, the first term is dominant and the system consists of a set of independent rotors. In the strong coupling limit the potential dominates and all vectors tend to align. As we increase $\lambda$ the excitation spectrum is also expected to change from a free rotor spectrum at small $\lambda$, to a spectrum containing three Goldstone modes, corresponding to the dynamical breaking of chiral symmetry, $O(4) \rightarrow O(3)$, at large $\lambda$.

This behavior can most easily be seen in the mean-field approximation. Let us assume that on average all the vectors align along the 4-axis. It can be shown that in that case we obtain [8] a nonlinear version of the Mathieu equation, which has a nontrivial solution (with broken symmetry) only for $\lambda D>3$, with a standard first-order phase transition at $\lambda$ $=3 / D$, where $D$ is the dimensionality of space. Below the phase transition point the wave function at each site is just the spherically symmetric $S$ wave of the free rotor, and above the phase transition we find a two-dimensional degeneracy, corresponding to positive and negative alignment with the 4-axis.

In the symmetric phase, one can ignore the one-body alignment, and concentrate on two-site correlations. We have investigated these processes using the normal coupled- cluster method [6], and have found that without the one-body term there is a termination point for one, two, and three dimensions. This has been interpreted as a signature of a phase transition. In one dimension this cannot be true, since the Coleman theorem forbids such a phase transition. This makes one distrust the other results as well. We show that the extended CCM is able to give much more reliable results.

In the extended coupled cluster method the bra and ket states are parametrized independently, corresponding to the use of a bi-orthogonal basis. We define two operators $\hat{S}$ and $\hat{S}^{\prime \prime}$, from which we we build up a variational functional $I$ which is the ground state expectation value of the Hamiltonian in a special state,

$$
I\left[S^{\prime \prime}, S\right]=\left\langle\Phi_{0}\left|e^{\hat{S}^{\prime \prime}} e^{-\hat{S}} H e^{\hat{S}}\right| \Phi_{0}\right\rangle=\langle\widetilde{\Psi}|H| \Psi\rangle,
$$

where $\left|\Phi_{0}\right\rangle$ is the model, or reference, state, that must be chosen separately. A key ingredient in the definition of the correlations is a set of generalized creation operators, that must be chosen with reference to the state $\left|\Phi_{0}\right\rangle$, i.e., the Hermitian conjugates of the creation operators annihilate $\left|\Phi_{0}\right\rangle$. In the symmetric phase we shall use the free-rotor vacuum, the product of $S$ waves at each lattice site, as reference state. It can be shown that the use of single-site operators is a way to construct the mean-field approximation. When the mean-field is zero, the lowest nontrivial correlations are two-site operators,

$$
\hat{S}=\sum_{\mathbf{i}, \mathbf{j}} \hat{S}_{\mathbf{i j}}
$$

where the sums run over the infinite lattice, and similarly for $\hat{S}^{\prime \prime}$. The operators $\hat{S}_{\mathrm{ij}}$ create a simultaneous excitation at the lattice sites $\mathbf{i}$ and $\mathbf{j}$. The precise definition of these operators is not crucial to the current discussion, and can be found in our previous work [6]. By going to the coordinate representation, it has been shown that specification of the operators $\hat{S}_{\mathrm{ij}}$ corresponds to specification of a single function $S\left(\cos \theta_{\mathrm{ij}}\right)$. We choose to work in this representation. In the numerical calculation we have to truncate up to a certain length of the relative distance between $\mathbf{i}$ and $\mathbf{j}$, but this can be investigated systematically by using various truncations and studying convergence. Both the bra- and ket-state correlations have to be truncated in the same manner to ensure the conservation of certain analytical properties of the variational principle underlying the method [7]. The functions $S\left(\cos \theta_{\mathrm{ij}}\right)$ can be expanded in Gegenbauer polynomials of the relative angle $\theta_{\mathbf{i j}}$ between the unit vectors at lattice sites $\mathbf{i}$ and $\mathbf{j}$, but the expansion converges so quickly that the results are essentially converged with the first five polynomials. Because of the translational symmetry of the lattice the correlation functions depend only on the relative vector $\mathbf{i}-\mathbf{j}$. For an optimal choice of $S$ and $S^{\prime \prime}$ the functional $I$ must be stationary with respect to independent variations of the bra and ket states. This leads to the nonlinear ECCM equations [7] 


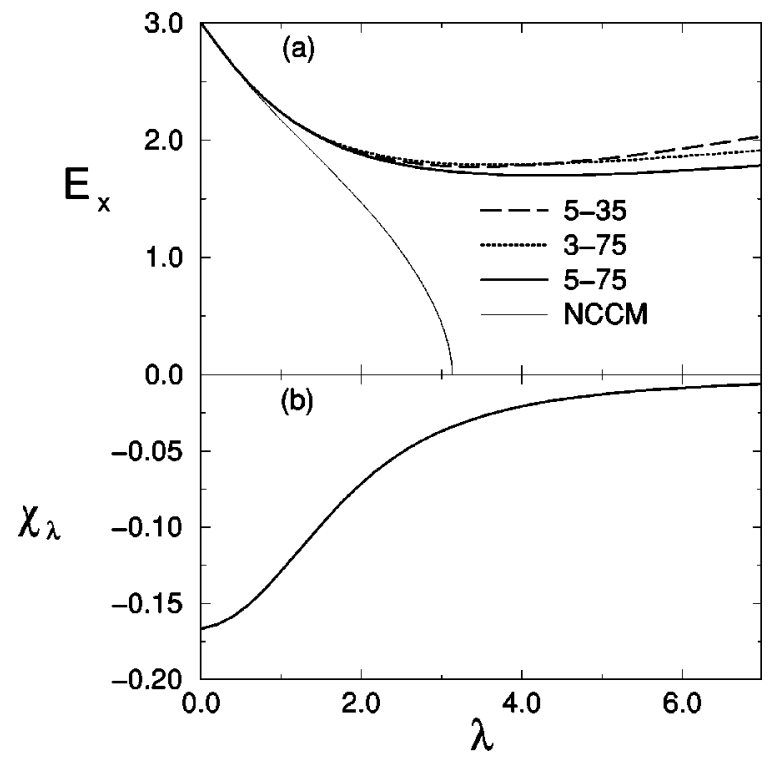

FIG. 1. (a) The lowest excitation energies and (b) the second derivative of the ground-state energy for the NLS model in (1 +1 ) dimensions. The labels $n-m$ refer to truncations at $n$ base functions and $m$ correlations.

$$
\frac{\delta I\left[\left\{S^{\prime \prime}, S\right\}\right]}{\delta S_{\mathbf{i}-\mathbf{j}}^{\prime \prime}}=0, \quad \frac{\delta I\left[\left\{S^{\prime \prime}, S\right\}\right]}{\delta S_{\mathbf{i}-\mathbf{j}}}=0 .
$$

Since the ket state is defined as an exponential it contains automatically all possible independent combinations of correlations on top of the (lowest-order) sum of independent correlations. This is a natural choice, since the correlation operators are combined in linked objects, such as in Goldstone's linked cluster theorem [9], and, indeed the coupled cluster method itself provides by far the easiest proof of this important theorem [10]. Due to the similarity transform exponential $e^{-\hat{S}}$ on the left-hand side of the Hamiltonian in the functional Eq. (3), I is a polynomial in the correlation functions. This has large advantages for high-order computer implementations to solve Eqs. (5).

We shall contrast the results of the extended coupled cluster method to those of the normal coupled cluster method (NCCM), where the bra state is parametrized linearly in $S^{\prime \prime}$,

$$
\langle\widetilde{\Psi}|=\left\langle\Phi_{0}\right|\left(1+\hat{S}^{\prime \prime}\right) e^{-\hat{S}}
$$

Therefore, in the NCCM, the overlap of the bra and ket states extends only as far as the correlation operator $\hat{S}^{\prime \prime}$. It has been shown in Ref. [7] that exponentiating $\hat{S}^{\prime \prime}$ extends the overlap over the whole lattice and also breaks certain symmetries of the functional which allows one to cross a phase transition, instead of breaking down at the phase transition point, which often occurs for the NCCM.

Using the time-dependent variational principle we can find low-lying excitations of the system by considering harmonic fluctuations about the ground state, also called the RPA approximation. Since we have the functional in an analytical form we are able to calculate the excitation energies relatively straightforwardly. (see Figs. 1 and 2.)

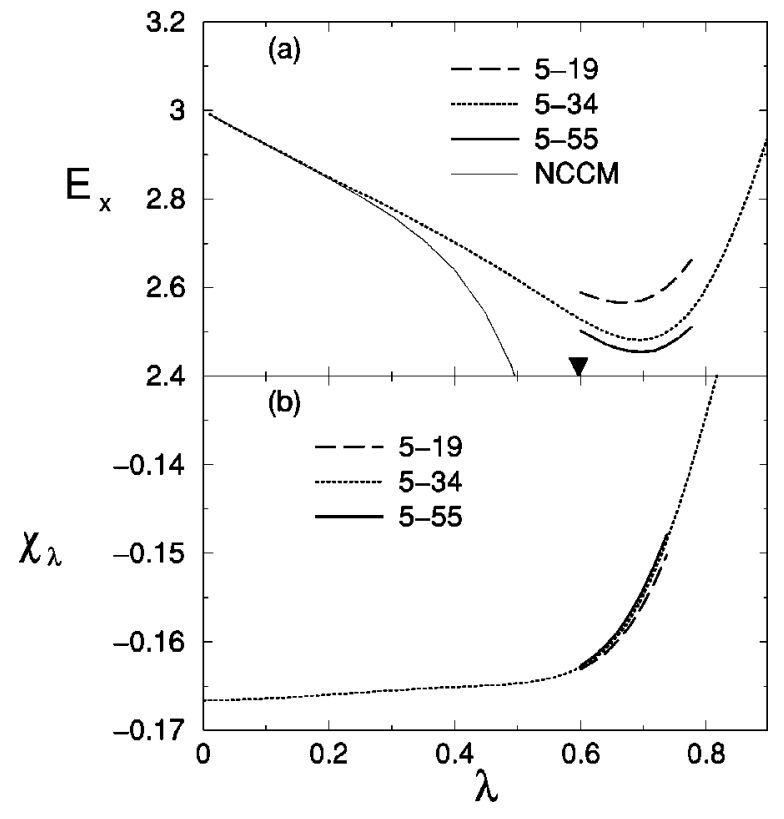

FIG. 2. (a) The lowest excitation energies, and (b) the second derivative of the ground-state energy for the NLS model in (3 $+1)$ dimensions. The triangle indicates the NCCM endpoint. The labels $n-m$ refer to truncations at $n$ base functions and $m$ correlations.

We have applied the ECCM to the NLS model on a (1 $+1)$ - and a $(3+1)$-dimensional cubic lattice. We have studied various truncations, and found that for the expansion of $S_{\mathrm{ij}}$ in Gegenbauer polynomials five such functions appear to be enough. For the truncation of the range of the functions $S$ we have proceeded as far as practicable, by including all correlations that fit within a cube of size $L$. For the $(1+1)$-dimensional case we can almost use arbitrarily large values of $L$, say including a hundred lattice sites. For the $(3+1)$-dimensional result the best calculation was limited to those correlations that fit inside a cube of length 5 .

For a linear chain the ground-state energy has converged completely with correlations which extend over up to 75 lattice sites. The susceptibility, the second-order derivative of the ground-state energy, changes smoothly with the coupling constant, indicating the absence of a transition region. This is confirmed by the excitation energy. The lowest excitation energy seems to have converged with five base functions and 75 correlations, although the global trend sets in at much lower orders. The mass gap remains finite. Clearly, these results are in agreement with the Coleman theorem and are a step beyond the mean-field, and NCCM, results.

For the cubic lattice the ground-state energy has more or less converged for five base functions and all 55 correlations that fit in a $5 \times 5 \times 5$ box. The susceptibility shows two regions: a linear, almost constant, susceptibility for the weakcoupling regime, and a steeper behavior for the strongcoupling regime. In low-order calculations the behavior between $\lambda \approx 0.6$ and $\lambda \approx 0.7$ changes drastically, while the trend on both sides outside this region is already predicted by low-order results. Even though the results do not allow us to make a definite statement about the nature of the transition, it seems weaker than a second-order phase transition. The ex- 
citation energies again confirm these results. Although the convergence is too slow to determine whether a massless mode appears, it dips at the phase transition and rises fast in the strong-coupling regime. Several low-lying excitation energies turn complex beyond the phase transition, indicating that excitations in this, distinctly different, phase are not properly described without the one-site correlations necessary to describe symmetry breaking. The absence of this phenomenon in $(1+1)$ dimensions is another indication that no phase transition occurs for this case.

The slow convergence in the transition region also indicates that many long-range correlations play an important role as is the case in a real phase transition. The absence of this behavior in $(1+1)$ dimensions is due to the special properties of the one-dimensional system that keeps the correlation length finite. The ECCM has the property that both the ket and bra states are expressed in linked-cluster coefficients. Therefore, the length over which ECCM correlation operators extend is a good indication of the length of the physical correlations.

Since we have a dual expansion, in the extent of the correlations, and in each correlation in a set of basis functions, we must look at the truncation systematics for both of these. For the $(1+1)$-dimensional system, beyond a minimum length of the correlations (about 30 lattice units), the results only depend on the number of basis functions, which indicates a finite, physical correlation length. The convergence with the number of basis functions is quite fast, indicating that the correlations are smooth functions of the relative angle, which indicates that the large number of correlations are essential to describe the phase transition properly since single correlation operators contribute only very little. This also holds for the $(3+1)$-dimensional system, but we never fully reach convergence with the range of the correlations. Both expansions seem to interplay in a complicated way in the results. However, the ground-state energies generally converge much faster than the excitation energies.

With the ECCM we find signatures for a phase transition of the $O(4)$ nonlinear sigma model in $(3+1)$ dimensions, which confirm our naive expectations. In $(1+1)$ dimensions the phase transition does not occur, as expected from Coleman's theorem. These results show clearly that ECCM is a proper many-body technique for the microscopic study of phase transitions and other critical phenomena in cases inaccessible with other methods, and we intend to use it in further studies.

We acknowledge support of a research grant (GR/ L22331) from the Engineering and Physical Sciences Research Council (EPSRC) of Great Britain.
[1] S. Coleman, Commun. Math. Phys. 31, 259 (1973).

[2] L.D. Faddeev and L.A. Takhtadzhyan, Phys. Lett. 85B, 375 (1981)

[3] C. Zeng, D.J.J. Farnell, and R.F. Bishop, J. Stat. Phys. 80, 327 (1998).

[4] S.E. Krüger et al., Phys. Rev. B 61, 14607 (2000).

[5] S.J. Baker, R.F. Bishop, and N.J. Davidson, Phys. Rev. D 53, 2610 (1996).

[6] N.E. Ligterink, N.R. Walet, and R.F. Bishop, Ann. Phys. (N.Y.) 267, 97 (1998).
[7] J.S. Arponen, Ann. Phys. (N.Y.) 151, 311 (1983); J.S. Arponen et al., Phys. Rev. A 36, 2519 (1987); 36, 2539 (1987); 37, 1065 (1988); J.S. Arponen and R.F. Bishop, Ann. Phys. (N.Y.) 207, 171 (1991); 227, 275 (1993); 227, 334 (1993).

[8] N. E. Ligterink, N. R. Walet, and R. F. Bishop (in preparation). [9] J. Goldstone, Proc. R. Soc. London, Ser. A 239, 267 (1957).

[10] R.F. Bishop, in Microscopic Quantum Many-Body Theories and Their Applications, edited by J. Navarro and A. Polls, Lecture Notes in Physics 510 (Springer, Berlin, 1998), p. 1. 